\title{
A Novel Percolation Theory for High Temperature Superconductors
}

\author{
E.V.L. de Mello, E.S. Caixeiro, and J.L. Gonzaléz \\ Departamento de Física, Universidade Federal Fluminense, \\ Av. Litorânia s/n, Niterói, R.J., 24210-340, Brazil
}

Received on 28 February, 2002

\begin{abstract}
We present a percolation theory for the high- $T_{c}$ oxides pseudogap and $T_{c}$ dependence on the hole level. The doping dependent inhomogeneous charge structure is modeled by a distribution which may represent the stripe morphology and yield a spatial distribution of local $T_{c}(r)$. The temperature onset of spatial dependent superconducting gap is identified with the vanishing of the pseudogap temperature $T^{*}$. The transition to a superconducting state corresponds to the percolation threshold among regions of different $T_{c}$. As a paradigm we use a Hubbard Hamiltonian with a mean field approximation to yield a doping and temperature dependent superconducting d-wave gap. We show here that this new approach reproduces the phase diagram, explains and gives new insights on several experimental features of high- $T_{c}$ oxides.
\end{abstract}

\section{Introduction}

High- $T_{c}$ oxides has been discovered fifteen years ago[1] but many of their important properties remains not well understood. Among these, the pseudogap phenomenon, that is, a discrete structure of the energy spectrum above $T_{c}$, identified by several different experiments[2], has its nature not yet been clarified. Such open problem has attracted a lot of experimental and theoretical effort because it is general belief that its solution is related to the understanding of the superconducting fundamental interaction.

The evidence of such energy gap above the superconducting phase is clearly demonstrated by tunneling $[3,4]$ and angle-resolved photoemission spectroscopy $[5,6]$ experiments. In the resistivity measurements its presence is seen by a decrease in the linear behavior below $T^{*}[7,8]$ and in the specific heat as a suppression in the linear coefficient $\gamma$ of the temperature[9].

There is also mounting experimental evidences that the hole doped inhomogeneity into the $\mathrm{CuO}$ planes, common to all cuprates, is directly related to the pseudogap phenomenon. In a given family, the underdoped compounds near the doping onset of superconductivity have the more inhomogeneous charge distributions and the larger $T^{*}$. As the doping level increases, the samples become more homogeneous while $T^{*}$ decreases[10, 11, 12]. For overdoped compounds $T^{*}$ disappears or becomes equal to $T_{c}$. The inhomogeneities were long supposed to be important in the studies of high temperature superconductors[13] but only after the discover of the spin-charge stripes[14], they have become a matter of systematic studies. In the spin-charge stripes scenario, regions of the $\mathrm{CuO}_{2}$ planes are hole-rich (the stripes) and others regions are hole-poor which fill the space between the stripes.

Recently, magnetic excitations as the vortex-like Nernst effect have been reported above $T_{c}[15]$ and a local Meissner state, which usually appears only in the superconducting phase, has been seen as a precursor to superconductivity[16]. Such inhomogeneous diamagnetic domains develop near $T^{*}$ and grow continuously as $T$ decreases towards $T_{c}$. Near $T_{c}$, the domains appear to percolate, according to Fig.3 from Iguchi et al.[16].

Based on all these experimental findings, about the pseudogap phenomenon, local charge inhomogeneities and a non percolative local Meissner state between $T^{*}$ and $T_{c}$, we propose a new scenario to explain the high $T_{c}$ superconductors phenomenology: due to the doping dependent charge inhomogeneities in a given compound with average charge density $\rho_{m}$, there is a distribution of local clusters with spatial dependent charge density $\rho(r)$, each with its superconducting transition temperature $T_{c}(r) . T^{*}$ is the largest of all $T_{c}(r)$. As the temperature falls below $T^{*}$, some clusters become superconducting, but they are surrounded by metallic and/or antiferromagnetic insulating domains and, consequently, the whole system is not a superconductor. The number of superconducting clusters increases as the temper- 
ature decreases, so the superconducting regions grow and, eventually, at a temperature $T_{c}$, they percolate and become able to hold a macroscopic dissipationless current. Exactly as the Meissner state domains shown in Fig.3 of Iguchi et al.[16]. Similar ideas were discussed by Ovchinnikov at al[17]. They were concerned mainly with the microscopic mechanism which leads to a distribution of $T_{c}(r)$ and its effect on the density of state.

In order to show that these ideas are able to make quantitative predictions and reproduce the high- $T_{c}$ oxides phase diagram, we have performed calculations on a Hubbard model and a gap equation is obtained within mean field approach to derive $T^{*} \times \rho$. Afterwards, using the percolation approach, we show that we can reproduce also the experimental values for the $\mathrm{Bi} 2212 T_{c} \times \rho$.

\section{The Charge Distribution}

The presence of microscopic charge inhomogeneities distribution in the $\mathrm{CuO}_{2}$ planes in a striped configuration is well documented[14]. It also implies in strong modifications for the local structure as the local $\mathrm{Cu}-\mathrm{O}$ bond length changes its size with the average local doping level[10, 11, 18].

The consequence of these intrinsic microscopic charge inhomogeneities distribution in the $\mathrm{CuO}_{2}$ planes, is the existence of two phases which are spontaneously created in the $\mathrm{CuO}_{2}$ planes; regions which are hole-rich form the stripes and others regions which are hole-poor (the antiferromagnetic insulator region) and are created between the charge-rich stripes (the metallic region). The exactly form of these charge distributions is not known and it is presently matter of research[10, 11, 18] but we can draw some insights from the STM/S results of Pan et al.[12]. Based on these STM/S data, we have chosen a combination of a Poisson and a Gaussian distribution. For a given compound with an average charge density $\rho_{m}$, the hole distribution $P$ is a function of the local hole density $\rho, P\left(\rho ; \rho_{m}\right)$. It is divided in two branches: The low density branch represents the insulating regions, and the high density one represents the metallic regions. As concerns the superconductivity, only the properties of the hole rich branch are important since the current flows through the metallic region. Such distribution may be given by:

$$
\begin{aligned}
P(\rho)= & \pm\left(\rho-\rho_{c}\right) \exp \left(-\left(\rho-\rho_{c}\right)^{2} / 2\left(\sigma_{ \pm}\right)^{2}\right) / \\
& \left(\sigma^{2}\right)\left(2-\exp \left(-(0.10)^{2} / 2\left(\sigma_{ \pm}\right)^{2}\right)\right)
\end{aligned}
$$

The plus sign is for the hole-rich $\left(\rho_{c} \approx \rho_{m}\right)$ for $\rho_{m} \leq \rho$, the minus to the hole-poor branch $\left(\rho_{c}=0.10\right)$ for $\rho \leq 0.10$ and $P(\rho)=0$ for $0.10 \leq \rho \leq \rho_{m}$.
From the experimental STM/S Gaussian histogram distribution for the local gap[12] in an optimally doped $\mathrm{Bi}_{2} \mathrm{Sr}_{2} \mathrm{CaCu}_{2} \mathrm{O}_{8+x}\left(\rho_{m} \approx 0.32\right)$ (see Fig.2d of Ref.12), we get that the half-width for the metallic branch is about half of the mean doping value, that is $2 \sigma_{+}=0.16$, since the local gap is proportional to the local hole density. Furthermore $\sigma_{+}$is related with the degree of inhomogeneities, and we estimate the $\sigma_{+}$values for the other compounds using the fact that it decreases with the hole density.

The distribution $P(\rho)$ for $\rho_{m}=0.32$ and $\sigma_{+}=0.08$ is shown in Fig.1a.. As a general feature for a family of compounds, based also in the experimental results of Pan et al.[12], for samples with $\rho_{m} \geq 0.5$, the charge distribution becomes a simple Gaussian centered at $\rho_{m}$ with $\sigma \leq 0.04$, which reflects the non-existence of the stripes phases for overdoped samples.

The values of $\sigma_{-}$for a given sample are chosen in order that percolation in the hole-rich branch occurs exactly at a given density $\rho_{p}$. Thus $T^{*}\left(\rho_{p}\right)$ is the maximum temperature which the system can percolate and which we identify as equal to $T_{c}\left(\rho_{m}\right)$. According to the percolation theory, the threshold of percolation occurs in a square lattice when $59 \%$ of the sites or bonds are filled[19]. Thus, we find the density where the holerich branch percolates integrating $\int P(\rho) d \rho$ from $\rho_{m}$ till the integral reaches the value of 0.59 , where we define $\rho_{p}$. Below $T_{c}\left(\rho_{m}\right)$ the superconducting region percolates and, consequently, it is able to hold a dissipationless supercurrent. To estimate $T_{c}\left(\rho_{m}\right)$ we need to calculate $T^{*}$ as function of $\rho$.

\section{The Phase Diagram}

To develop the dynamics of the hole-type carriers in the $\mathrm{CuO}_{2}$ planes, we adopt a two dimension extended Hubbard Hamiltonian in a square lattice of lattice parameter $a$

$$
\begin{gathered}
H=-\sum_{\ll i j \gg \sigma} t_{i j} c_{i \sigma}^{\dagger} c_{j \sigma}+U \sum_{i} n_{i \uparrow} n_{i \downarrow} \\
+\sum_{<i j>\sigma \sigma^{\prime}} V_{i j} c_{i \sigma}^{\dagger} c_{j \sigma^{\prime}}^{\dagger} c_{j \sigma^{\prime}} c_{i \sigma}
\end{gathered}
$$

where $t_{i j}$ is the hopping integral between sites $i$ and $j$; $U$ is the Coulomb on-site correlated repulsion and $V_{i j}$ is the a phenomenological attractive interaction between nearest-neighbor sites.

Using a BCS-type mean-field approximation to develop Eq.(2) in the momentum space, one obtains the self-consistent gap equation, at finite tempera- 
tures $[20,21,22]$

$$
\Delta_{\mathbf{k}}=-\sum_{\mathbf{k}^{\prime}} V_{\mathbf{k} \mathbf{k}^{\prime}} \frac{\Delta_{\mathbf{k}^{\prime}}}{2 E_{\mathbf{k}^{\prime}}} \tanh \frac{E_{\mathbf{k}^{\prime}}}{2 k_{B} T},
$$

with $E_{\mathbf{k}}=\sqrt{\varepsilon_{\mathbf{k}}^{2}+\Delta_{\mathbf{k}}^{2}}$, which contains the potential $V_{\mathbf{k k}^{\prime}}$. For a d-wave order parameter, $U$ is summed out of the gap equation and the amplitude of the attractive potential $V$ is the only unknown variable which becomes an adjustable parameter[22, 23]. $\varepsilon_{\mathbf{k}}$ is a dispersion relation. The hole density and the gap equation, are solved self-consistently for a d-wave order parameter[22, 23].

In Fig.1b we plot the temperatures of vanishing gap $T^{*}$ as function of $\rho$, derived with the above equations. Here we use $V=-0.150 \mathrm{eV}$ which reproduces well the experimental values of $T^{*} \times \rho$ for the Bi2212 system[7]. The hopping parameters are within $15 \%$ to those taken from ARPES measurements [24] with a nearest neighbor hopping $t_{i j}=0.12 \mathrm{eV}\left(t_{i j}=0.147 \mathrm{eV}\right.$ in ref.24) and further hopping parameters up to fifth neighbor. The particular form of the $T^{*}$ curve depends on the values of these hoppings. Notice that the density of holes has a factor of 2 with respect to the values given by Ref.[7], but in agreement with ref.[21] as it is more appropriate for the Bi2212 system[25].

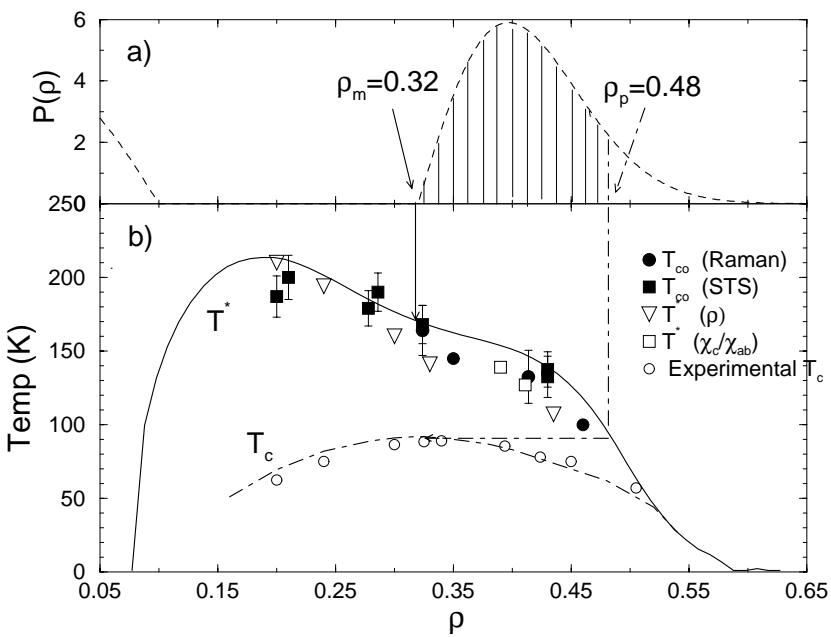

Figure 1. 1a) The probability distribution for the optimal compound with $\rho_{m}=0.32$. 1b) The calculated onset of vanishing gap $T^{*}(\rho)$. The thick arrow shows $T^{*}\left(\rho_{m}\right)$ and the dot-dash arrow shows how $T_{c}\left(\rho_{m}\right)$ is found. The experimental points and the symbols are taking from Ref.[7]

The $T^{*} \times \rho_{m}$ and $T_{c} \times \rho_{m}$ diagrams are obtained as follows: A compound with an average hole content higher than the onset of superconductivity for Bi2212, that is, $\rho_{m} \geq 0.15$, has its metallic branch with local densities $\rho(r)$ varying from $\rho_{m}$ to higher values. In this range, $T^{*}$ is a decreasing function of $\rho$. Therefore for temperatures above $T^{*}\left(\rho_{m}\right)$, there is neither any gap nor any superconducting region and $T^{*}\left(\rho_{m}\right)$ marks the appearance of a gap in the sample. That is why $T^{*}\left(\rho_{m}\right)$ is the $T^{*}$ of the system. On the other hand, $T_{c}\left(\rho_{m}\right)$ is calculating integrating $\int P(\rho) d \rho$ from $\rho_{m}$ till the integral reaches the value of 0.59 (the integrated region is marked with vertical lines in Fig.1a), where we define $\rho_{p}$. Only at $T \leq T^{*}\left(\rho_{p}\right)$ (see Fig.1a for $\rho_{p}=0.48$ ) the compound may hold a superconducting current since the superconducting regions percolates through the sample. Thus $T^{*}\left(\rho_{p}\right)$ is the percolating threshold and it is equal to $T_{c}\left(\rho_{m}\right)$ as one can see following the dot-dashed line in Fig.1a and 1b).

It is worthwhile to mention that the percolating approach could also be used with others methods to calculating $T^{*} \times \rho_{m}$, like, for instance, the one which uses the Hubbard-Holstein Hamiltonian[27].

\section{Discussion}

The fact that $T^{*}$ decreases continuously with $\rho$ in the superconducting region $\left(\rho_{m}>0.15\right)$, as seen in Fig.1b, is very suggestive and it is in agreement with the early ideas regarding a phonon mediated superconducting interactions: Materials whose vibrating atoms interact strongly with the electrons, and are poor metals, should become superconductors at higher temperatures than those good metals, whose atoms interact weakly with electrons[26]. For cuprates, as the doping level of the samples increases, it is well known that the compounds change from very poor metals in the normal phase to very good metals with typical Fermi liquid behavior for overdoped samples. Since $T^{*} \times \rho_{m}$ is a decreasing function of doping for any cuprate family beyond the onset of superconductivity, such behavior may be a strong indication of the phononic superconducting interaction.

There are several observations and measurements that can be well explained within the percolating approach, we will discuss here only a few examples.

1-Harris et al.[28], through ARPES measurements, have reported the anomalous behavior of $\Delta_{0}\left(\rho_{m}\right)$ which decreases steadily with the doping $\rho_{m}$ although $T_{c}$ increases by a factor of 2 for their underdoped samples. In the overdoped region, since $T_{c}$ also decreases, the behavior is the expected conventional proportionality. It is well known that superconductors have a constant value for the ratio $2 \Delta_{0} / k_{B} T_{c}$, being 3.75 for usual isotropic order parameter and 4.18 for $d_{x^{2}-y^{2}}$ solution[29].

At low temperature, since the superconducting region percolates through different regions, each with a given $\Delta_{0}(r)$, tunneling and ARPES experiments detect the largest gap present in the compound. Consequently, $\Delta_{0}\left(\rho_{m}\right)$ must be correlated with the onset of vanishing gap $T^{*}\left(\rho_{m}\right)$ which is the largest superconducting temperature in the sample, and not with $T_{c}\left(\rho_{m}\right)$. As we show in Fig.2, correlating the values for $T^{*}\left(\rho_{m}\right)$ with $\Delta_{0}\left(\rho_{m}\right)$, we are able to give a reasonable fit for the data 
of Harris et al.[28] on $D y-B S C C O$ and explains the different energy scales pointed out by Harris et al. and several others authors.

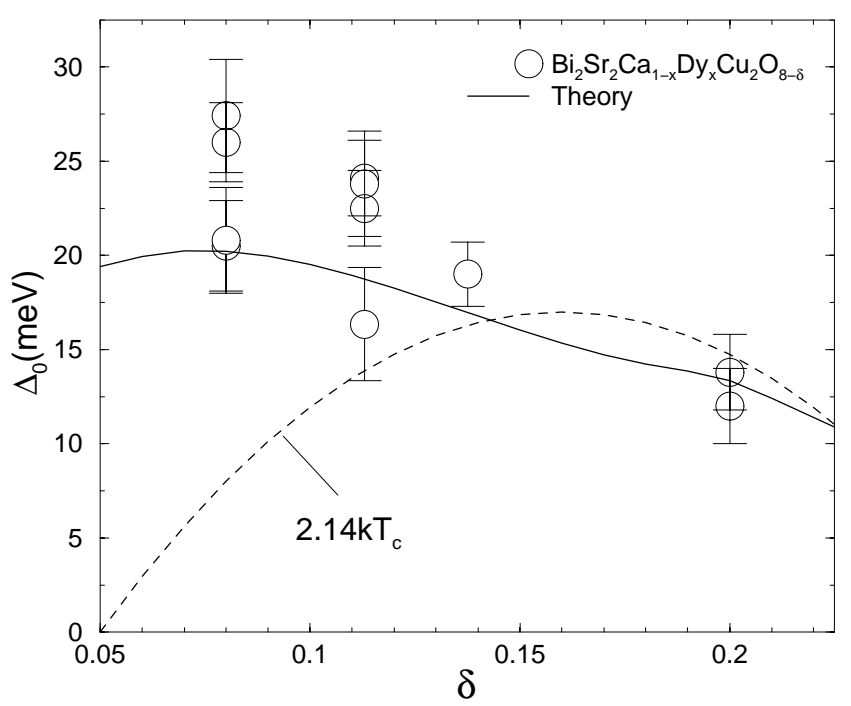

Figure 2. The zero temperature gap for 9 samples as measured by Harris et al[28] and our calculations.

2- The resistivity for underdoped and optimum doped compounds deviates at $T^{*}$ from the linear behavior and falls faster as the temperature[2, 7] decreases. This behavior can be understood by our model, with the increasing of superconducting cluster numbers and size, as the temperatures decreases below $T^{*}$. Each superconducting cluster produces a short circuit which decreases the resistivity below the linear behavior between $T^{*}$ and $T_{c}$.

3- As mentioned in the introduction, the existence of superconducting cluster between $T^{*}$ and $T_{c}$ easily explains the appearance of local diamagnetic or Meissner domains, and, if there is a temperature gradient in the sample, the local flux flows and produces the dynamic flux flow state[15].

4- Another important consequence is that the pairing mechanism must be investigated by experiments performed mainly at $T^{*}$. A such experiment was accomplished by Rubio Temprano et al.[30], which measured a large isotope effect associated with $T^{*}$ and an almost negligible isotopic effect associated with $T_{c}$ in the slightly underdoped $\mathrm{HoBa}_{2} \mathrm{Cu}_{4} \mathrm{O}_{8}$ compound. The results strongly support the fact that electronphonon induced effects are present in the superconducting mechanism associated with $T^{*}$.

\section{Conclusions}

We have demonstrated that the percolating approach for an inhomogeneous charge distribution on the $\mathrm{CuO}_{2}$ planes provides new physical explanations for many experiments performed on high- $T_{c}$ oxides and quantitative results for their phase diagram.
Contrary to some current trends, in which $T_{c}$ is regarded as a phase coherence temperature and the existence of a gap phase without coherence between $T_{c}$ and $T^{*}$, in our approach, $T_{c}$ is a percolating temperature for different regions which, due to the inhomogeneities, possess different local superconducting transition $T^{*}(r)$. Similarly, instead of having a superconducting gap $\Delta_{s c}$ and an excitation gap $\Delta$ associated with $T^{*}$, we have a distribution of locally dependent $\Delta_{s c}(r)$.

The percolation method introduced here can be applied in any cuprate and yields also several new implications which will be discussed elsewhere, but one of the most interesting is that one can search for materials with very large $T_{c}$ 's if a better control of the local doping level is achieved.

Financial support of $\mathrm{CNPq}$ and FAPERJ is gratefully acknowledged. JLG thanks CLAF for a CLAF/CNPq pos-doctoral fellowship.

\section{References}

[1] J.G. Bednorz and K.A. Müller, Z. Phys. 64, 189 (1986).

[2] T. Timusk and B. Statt, Rep. Prog. Phys. 62, 61 (1999).

[3] C. Renner, B. Revaz, J.-Y Genoud, K. Kadowaki, and O. Fischer, Phys. Rev. Lett. 80, 149 (1998).

[4] M. Suzuki, T. Watanabe, and A. Matsuda, Phys. Rev. Lett. 82, 5365 (1999).

[5] Z-X. Shen and D. S. Dassau, Phys. Rep. 253, 1 (1995).

[6] H. Ding, T. Yokkoya, J.C. Campuzano, T. Takahashi, M. Randeria, M.R Norman, T. Mochiku, K. Kadowaki, and J. Giapintzaki, Nature, 382, 511996

[7] M. Oda, N. Momono, and M. Ido, Supercond. Sci. Technol. 13, R139, (2000).

[8] H. Takagi, B. Batlogg, H.L. Kao, R.J. Cava, J.J. Krajewski, and W.F. Peck, Phys. Rev. Lett. 62, 2975 (1992).

[9] J. Loram, K.A. Mirza, J.R. Cooper, and J.L. Tallon, Physica C282-287, 1405 (1997).

[10] S.J.L. Billinge, E.S. Bozin, M. Gutmann, and H. Takagi, J. Supercond., 2306 (2000), Proceedings of the Conf. "Major Trends in Superconductivity in New Milenium", and cond-mat 0005032.

[11] E.S. Bozin, G.H. Kwei, H. Takagi, and S.J.L. Billinge, Phys. Rev. Lett. 84, 5856, (2000).

[12] S.H. Pan, J.P. ONeal, R.L. Badzey, C. Chamon, H. Ding, J.R. Engelbrecht, Z. Wang. H. Eisaki, S. Uchida, A.K. Gupta, K.W. Ng, W.W. Hudson, K.M. Lang, and J.C. Davis, Nature 413, 282 (2001) and condmat/0107347.

[13] T. Egami and S.J.L. Billinge, in "Physical Properties of High-Temperatures Superconductors V" edited by D.M. Greensberg, World Scientific, Singapure 1996), p. 265. 
[14] J.M.Traquada, B.J. Sternlieb, J.D. Axe, Y. Nakamura, and S. Uchida, Nature (London), 375, 561 (1995).

[15] Z.A. Xu, N.P. Ong, Y. Wang, T. Kakeshita, and S. Uchida, Nature 406, 486 (2000).

[16] I. Iguchi, I. Yamaguchi, and A. Sugimoto, Nature, 412 , 420 (2001).

[17] Yu.N. Ovchinnikov, S.A. Wolf, and V.Z. Krezin, Phys. Rev. B63, 6452, (2001), and Physica C341-348, 103, (2000)

[18] T. Egami, Proc. of the New3SC International Conference, to be published in Physica C.

[19] D.F. Stauffer and A. Aharony, Introduction to Percolation Theory, Taylor \& Francis Ed., London, 1994.

[20] E.V.L. de Mello, Physica C 259, 109 (1996).

[21] G.G.N. Angilella, R. Pucci, and F. Siringo, Phys. Rev. B 54, 15471 (1996).

[22] E.S. Caixeiro, and E.V.L. de Mello, Physica C 353 , 103 (2001).

[23] E. S. Caixeiro, and E.V.L. de Mello, submitted to the J. Phys. CM.

[24] M.R. Norman, M. Randeria, H. Ding, J.C. Campuzano, and A.F Bellman, Phys. Rev. B52, 615 (1995).
[25] P. Konsin, N. Kristoffel, and B. Sorkin, J. Phys. C.M. 10, 6533 (1998)

[26] B.T. Matthias, Superconductivity, Scientific American, 92, November of 1957.

[27] C. Di Castro, M. Grilli, and S. Caprara, condmat/0109319.

[28] J.M. Harris, Z.H. Shen, P.J. White, D.S. Marshall, M.C. Schabel, J.N. Eckstein, and I. Bozovic, Phys. Rev. B54, R15665 (1996).

[29] H. Won and K. Maki, Phys. Rev. B49, 1397 (1994).

[30] D. Rubio Temprano, J. Mesot, S. Janssen, K. Conder, A. Furrer, H. Mutka, and K.A. Müller, Phys. Rev. Lett. 84, 1990 (2000).

[31] M.T.D. Orlando, A.G Cunha, E.V.L. de Mello, H. Belich, E. Baggio-Saitovich, A. Sin, X. Obradors, T. Burghardt, and A. Eichler, Phys. Rev. B61, 15454 (2000).

[32] J.L. Gonzaléz, M.T.D. Orlando, E.S. Yugue, E.V.L. de Mello, and E. Baggio-Saitovich, Phys. Rev. B63, 54516 (2001). 